f. med. Genet. (1965). 2, 47 .

\title{
Culture of Peripheral Blood Leucocytes for Chromosome Analysis using a Few Drops of Whole Blood
}

\author{
J. A. DARTNALL and J. E. GRAY \\ From the University of Newcastle upon Tyne
}

A number of modifications of Moorhead's original technique for culturing peripheral leucocytes have been described, enabling cultures to be grown from a few drops of blood (Arakaki and Sparkes, 1963; Edwards, 1962; Robinson, Bishun, Rashad, and Morton, 1964). They require the use of stored calf, chick, or human serum, and this has obvious disadvantages for the small laboratory. We have, therefore, recently been using a technique for culturing small amounts of blood from a baby using plasma from a parent or other convenient adult.

Three or four drops of blood can be collected from the baby from a heel prick. They can be conveniently collected into a disposable syringe containing $0.5 \mathrm{ml}$. Glaxo 199 medium and 100 units heparin. If the mixture is transferred to a bottle it can be kept for up to an hour at least.

A Io-ml. blood sample is taken from the parent, and some of this is used to set up a culture in the usual way. The residue is then spun quickly, until I. $5 \mathrm{ml}$. clear plasma can be removed. To ensure

Received November 19, 1964. that all parental cells have been removed this is then mixed with $5 \mathrm{ml}$. 199 and the mixture respun. The top $4 \mathrm{ml}$. of this are used (with P.H.A.) as a culture medium for the baby's blood. By using blood from the parent opposite in sex to the baby any parental cells in the resulting preparations should be easily recognized, but no example of this has been found.

The cultures are harvested in the usual way. We have used the method six times and obtained clear preparations on each occasion, and were able to analyse the karyotype of 20 cells in each case without difficulty. We have found the method useful in the newborn when for some reason (e.g. inexperience of the house physician or a small baby in an incubator) venepuncture is likely to be difficult.

\section{REFERENCES}

Arakaki, D. T., and Sparkes, R. S. (1963). Microtechnique for culturing leukocytes from whole blood. Cytogenetics, 2, 57.

Edwards, J. H. (1962). Chromosome analysis from capillary blood. ibid., $1,90$.

Robinson, J. S., Bishun, N. P., Rashad, M. N., and Morton, W. R. M. (1964). Chromosome analysis from capillary blood. Lancet, I, 328. 